\title{
Dendritic transmitter release and analog computation in mitral
}

\section{cells}

\author{
Jason B Castro*1,2 and Nathaniel N Urban ${ }^{1,2,3}$
}

Address: ${ }^{1}$ Center for Neuroscience, University of Pittsburgh, Pittsburgh, PA 15213, USA, ${ }^{2}$ Center for the Neural Basis of Cognition, Carnegie Mellon University, Pittsburgh PA 15216, USA and ${ }^{3}$ Department of Biological Sciences, Carnegie Mellon University, Pittsburgh PA 15216, USA

Email: Jason B Castro* - jasonc@pitt.edu

* Corresponding author

from Sixteenth Annual Computational Neuroscience Meeting: CNS*2007

Toronto, Canada. 7-12 July 2007

Published: 6 July 2007

BMC Neuroscience 2007, 8(Suppl 2):PI27 doi:I0.1 I86/I47|-2202-8-S2-PI27

() 2007 Castro and Urban; licensee BioMed Central Ltd.

Mitral cells of the accessory olfactory bulb contain between 2-10 principal dendrites, each of which terminates in a densely branched tuft contained in the glomerular layer. This multi-tufted morphology has led to the proposal that tufts may serve as local subthreshold processing stations that function independently of one another and in relative isolation from the soma. Consistent with this, we have found that focal synaptic stimulation of individual tufts results in regenerative calcium spikes restricted to the tuft $(n=7)$. In addition, tufts are closely associated with local interneurons called periglomerular (PG) cells, with which they from reciprocal dendrodendritic synapses. In experiments in which mitral cells were patched and filled with the calcium indicator Calcium Orange, we found a strong covariation between tuft calcium influx and inhibition received by mitral cells $(n=8)$, suggesting that tufts may self regulate their excitability via subthreshold synaptic mechanisms. To test this more directly, we first patched mitral cells with normal internal and evoked inhibition by activation of tuft spikes via afferent inputs. We then repatched with internal containing $1 \mathrm{mM}$ BAPTA to buffer intracellular calcium concentration, which should reduce release from the mitral cell $(n=5)$. The magnitude of inhibition received by the mitral cell decreased by $75 \% \pm 27 \%$ within 2 minutes of BAPTA repatch. In a final set of experiments, we performed paired recordings of mitral and PG cells and found that synaptically evoked tuft calcium transients were highly correlated with the amplitude of EPSPs recorded in PG cells. Moreover, the synaptic input to PG cells was reduced by hyperpolarizing the mitral cell.

The above data motivate a model of synaptic integration in AOB mitral cells in which each tuft acts as an independent processing station capable of providing synaptic output to local interneurons. We are currently exploring the computational consequences of this phenomenon by modeling AOB mitral cells as binary classifiers and exploring how dendritic transmitter release contributes to their ability to discriminate among analog patterns. 